\title{
Breast Cancer Presenting as Sudden Bilateral Hearing Loss: Case Report
}

\author{
Sandra Geros ${ }^{\mathrm{a}, \mathrm{b}}$, Teresa Bernardo ${ }^{\mathrm{a}}$, Eugenia Castro $^{\mathrm{a}}$, \\ Fernanda Castro ${ }^{a}$, Artur Conde ${ }^{a}$
}

\begin{abstract}
Sudden sensorineural hearing loss is defined as a rapid onset hearing impairment occurring over a 72 -h period, equal or greater than $30 \mathrm{~dB}$ in three consecutive frequencies. The annual incidence is 5-20 cases per 100,000 , most being unilateral. Simultaneous bilateral involvement is rare, occurring in $1-2 \%$ of all cases. This is the first report of bilateral involvement as the presenting symptom of breast cancer diagnosed 1 year later. The authors present a case of a female patient, 63-year-old, who appeared in the emergency department with sudden bilateral deafness as the only symptom. The examination showed no further alterations. Additional studies were performed, and the patient was admitted in the hospital for monitoring, therapy and etiologic investigation. After investigation, we concluded this was a rare case of severe bilateral sensorineural hearing loss associated with occult carcinoma of breast, diagnosed later. This is of relevance not only to otolaryngologists, but also to clinicians who may deal with similar complaints, because only a third will have an underlying etiology that is diagnosed along the way. Sudden hearing loss is an ear emergence, in which fast evaluation by a specialist or someone who may recognize the complaints significantly improves the prognosis for hearing recovery.
\end{abstract}

Keywords: Acute sensorineural hearing loss; Breast carcinoma; Hearing disorders; Inner ear pathology; Pure tone audiometry; Sudden deafness; Sudden hearing loss

\section{Introduction}

Sudden sensorineural hearing loss (SSNHL) is defined as a

Manuscript accepted for publication February 11, 2014

${ }^{\text {a } C e n t r o ~ H o s p i t a l a r ~ d e ~ V i l a ~ N o v a ~ d e ~ G a i a, ~ E s p i n h o, ~ R u a ~ C o n c e i c a o ~}$ Fernandes, 4434-502 VN Gaia, Portugal

${ }^{\mathrm{b}}$ Corresponding author: Sandra Geros, ENT Department, Centro Hospitalar de Vila Nova de Gaia, Espinho, Rua Conceicao Fernandes, 4434-502 VN Gaia, Portugal. Email: sandra.geros@gmail.com

doi: http://dx.doi.org/10.14740/jmc1688w rapid onset hearing impairment occurring over a 72-h period, equal or greater than $30 \mathrm{~dB}$ in three consecutive frequencies in pure tone audiometry. The annual incidence can go from 5 to 20 cases per 100,000 , mostly unilateral, whose prognosis for hearing recovery is generally good [1]. Simultaneous bilateral involvement is extremely rare, occurring in about $1-2 \%$ of all cases [2]. Non-idiopathic causes of SSNHL must be identified and addressed, and the most frequent are vestibular schwannoma, stroke and malignancy [3]. But still up to $90 \%$ of SSNHL are idiopathic at presentation [4]. Long-term follow-up is recommended as some of these patients may have an underlying cause that may not be evident at initial presentation [5]. SSNHL is a frightening symptom that often prompts an urgent management, which may improve hearing recovery and patient quality of life. That is why early recognition of signs and symptoms is so important.

Prognosis for recovery depends on several factors, including presence of vertigo at onset, patient's age, degree of hearing loss and time between onset and treatment [6].

Bilateral SSNHL as manifestation of occult breast cancer is a very rare clinical event with poor prognosis. In this report, we describe the case of a middle-aged woman with this initial complaint who was diagnosed with breast cancer 1 year after.

\section{Case Report}

A 63-year-old Caucasian woman with known cardiovascular risk factors (hypertension, diabetes mellitus type 2 and dyslipidemia) was under medication. She had a past history of "slight right hearing loss" since childhood (unknown etiology, according to the patient), with no interference in daily activities. She was admitted to the Emergency Department of Centro Hospitalar de Vila Nova de Gaia, Espinho with bilateral sudden deafness with onset on the same day, associated with bilateral tinnitus, without vertigo or other complaints. There was no report of any family history of deafness, personal history of virusal syndrome, acoustic trauma, physical exertion, introduction or replacement of any of the usual medicines. The examination showed bilateral severe sensorineural hearing loss suggested in acumetry without 


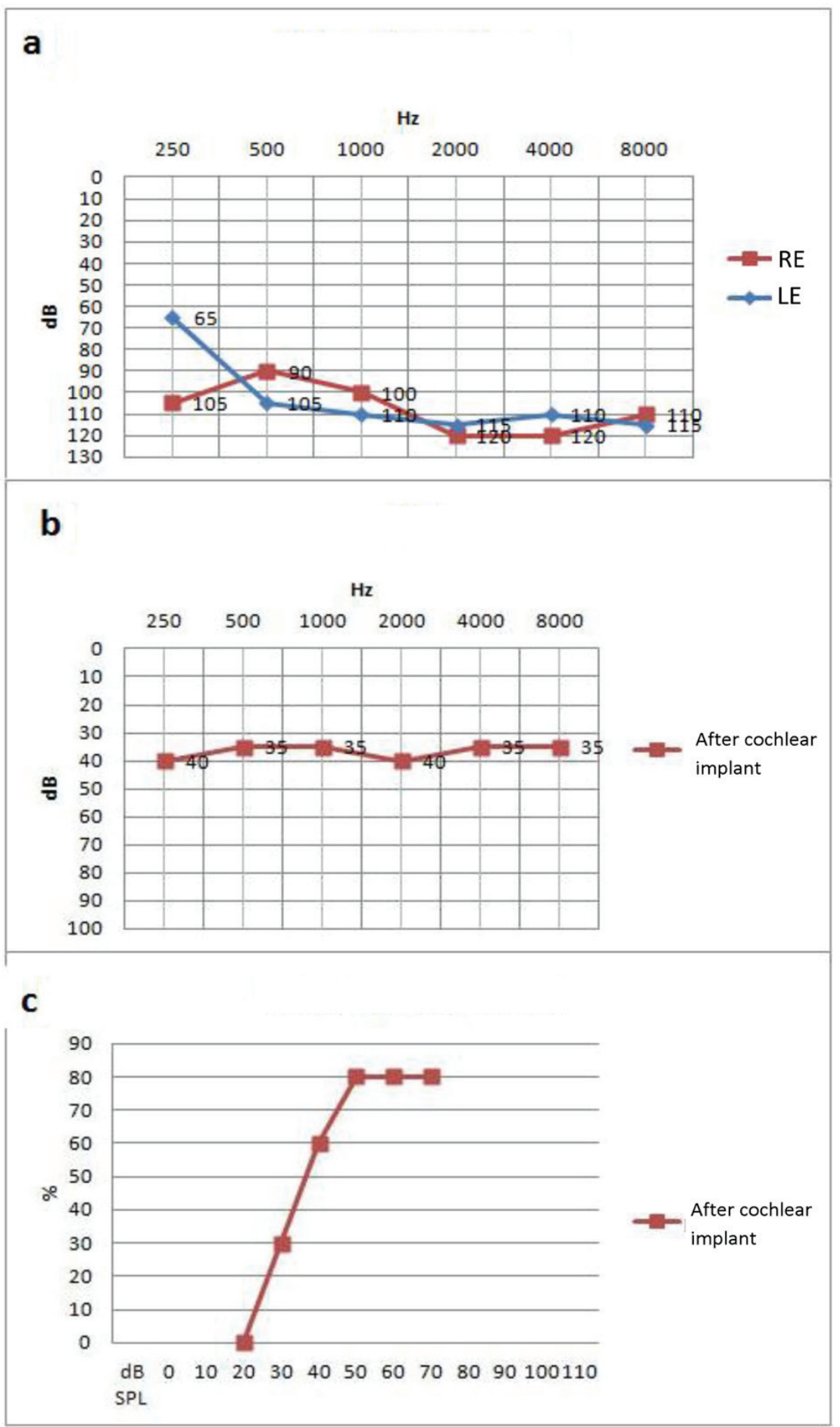

Figure 1. Audiogram before and after cochlear implant. (a) Pre-implant tone audiogram (TA), with bilateral profound sensorineural hearing loss, with slight improvement at low frequencies in the left ear (LE) after hyperbaric oxygen therapy (HBOT). (b) Tone. (c) Vocal audiogram after cochlear implant.

any other changes, including normal otoscopy. She was admitted to the Otolaryngology Department from the same hospital for monitoring, optimal therapy (including systemic corticosteroids) and etiologic investigation.
Pure tone audiometry revealed a bilateral profound sensorineural hearing loss. Auditory brainstem response (ABR) had no normal waveforms. Full blood count, urea and electrolytes, liver functions tests, clotting profile and 
tumor markers had no alterations. The screening for serological, autoimmune, or vascular disorders was conducted with negative results. Chest radiograph and brain magnetic resonance imaging (MRI) were normal. At day 11, she began intratympanic corticosteroid, early suspended because of adverse side effects (headache and worsening of tinnitus). At day 16, she began hyperbaric oxygen therapy (HBOT) and completed 20 daily sessions, with progressive clinical and objective (decreased intensity of tinnitus and slight audiometric) improvement (Fig. 1a).

Still in hospital, the patient had an episode of hematic discharge from right nipple (according to her with similar episodes at home). Physical examination and preliminary mammogram showed up inconclusive (breast imaging reporting and data system 0 - BI-RADS 0). Investigation continued after patient's discharge, with galactography and breast ultrasound, revealing a slight right retromamilar ductal ectasia. Cytology showed a hyperplastic epithelial fibrosis, whose histology of surgical excision revealed an invasive ductal carcinoma grade 2. Right mastectomy with sentinel node was performed, whose extemporaneous examination revealed disease-free margins and absence of metastatic lymph nodes.

Because of small improvement on hearing in follow-up, she was proposed and submitted to cochlear implantation (left-sided), with subsequent multidisciplinary rehabilitative evaluations. Postoperative audiometric evaluation showed significant improvement of hearing loss (Fig. 1b, c).

\section{Discussion}

SSNHL's etiology is identified in only $10-15 \%$ of patients at presentation. In up to one-third of cases, it may be identified only after long-term follow-up evaluations [7]. In a meta-analysis from Chau et al, the etiology of SSNHL was predominantly idiopathic (71\%), followed by infectious $(12.8 \%)$, otologic $(4.7 \%)$, trauma $(4.2 \%)$, vascular $(2.8 \%)$, neoplastic (2.3\%) or others (2.2\%) [8]. In March 2012, a clinical practice guideline was published by the American Academy of Otolaryngology-Head and Neck Surgery, not only to provide clinicians with evidence-based recommendations in evaluating patients with SSNHL but also to improve diagnostic accuracy [9].

When bilateral involvement is detected, the strong suspicion of an underlying systemic condition has to be investigated. In a review of medical literature, we found case reports associating bilateral SSNHL and neoplastic diseases, such as multiple myeloma, gastric, pancreatic and breast cancer. All related with central nervous system manifestations of metastatic disease. An example is a case report of bilateral SSNHL as the primary manifestation of meningeal carcinomatosis from occult breast carcinoma, diagnosed early after [10]. Another possible etiologic factor could be the association between malignancy and a hypercoagulated state. It is thought that tumor cells activate the blood coagulation system either by directly stimulating thrombin formation or by inducing mononuclear cells to synthesize procoagulants such as tissue factors, prothrombin activators and platelet aggregation or activate endothelial cells by certain cytokines (TNF and IL-1) to generate substances that stimulate the production of coagulation tissue factors [11].

In this particular case, the initial symptoms and the investigation did exclude most of the possible and common etiologies described in literature, including a hypercoagulated state. However, a careful follow-up and attention to the patient's signs took us to the final diagnosis of an occult breast carcinoma without any evidence of metastatic disease (confirmed not only with radiologic imaging but also histologically in sentinel node biopsy), which presented first as hematic mammillary discharge neglected by the patient and months later with bilateral SSNHL.

\section{Conclusion}

After all the research developed, we concluded this was a very rare case of severe bilateral SSNHL associated with breast cancer, diagnosed almost 1 year after initial symptoms. This is of particular relevance not only to ear, nose and throat practitioners, but also to other clinicians who may deal with similar complaints, because only a third of cases of SSNHL will have an underlying etiology that is diagnosed along the way. Although the vast majority of cases are labeled as idiopathic, it may represent the first sign of a serious underlying pathology, especially when there is bilateral involvement, so a thorough investigation and long-term monitoring can make the difference, like in this particular case report.

Sudden hearing loss is considered an ear emergence, in which fast and efficient evaluation of the patient by a specialist, significantly improves the prognosis for hearing recovery.

\section{Acknowledgements}

I would like to thank Dra. Sandra Alves who contributed towards the article by revising it critically for important intellectual content.

\section{Grant}

None.

\section{Conflict of Interests}

None. 


\section{References}

1. Korpinar S, Alkan Z, Yigit O, Gor AP, Toklu AS, Cakir $\mathrm{B}$, Soyuyuce OG, et al. Factors influencing the outcome of idiopathic sudden sensorineural hearing loss treated with hyperbaric oxygen therapy. Eur Arch Otorhinolaryngol. 2011;268(1):41-47.

2. Mathur N. Sudden hearing loss. Medscape Reference. Mar 2012;1-6. Available at http://emedicine.medscape. com/article/856313-overview. Access on April 24th 2012.

3. Saunders JE, Luxford WM, Devgan KK, Fetterman BL. Sudden hearing loss in acoustic neuroma patients. Otolaryngol Head Neck Surg. 1995;113(1):23-31.

4. Rauch SD. Clinical practice. Idiopathic sudden sensorineural hearing loss. N Engl J Med. 2008;359(8):833-840.

5. Penido Nde O, Ramos HV, Barros FA, Cruz OL, Toledo RN. Clinical, etiological and progression factors of hearing in sudden deafness. Braz J Otorhinolaryngol. 2005;71(5):633-638.

6. Conlin AE, Parnes LS. Treatment of sudden sensorineu- ral hearing loss: I. A systematic review. Arch Otolaryngol Head Neck Surg. 2007;133(6):573-581.

7. Haynes DS, O’Malley M, Cohen S, Watford K, Labadie RF. Intratympanic dexamethasone for sudden sensorineural hearing loss after failure of systemic therapy. Laryngoscope. 2007;117(1):3-15.

8. Chau JK, Lin JR, Atashband S, Irvine RA, Westerberg BD. Systematic review of the evidence for the etiology of adult sudden sensorineural hearing loss. Laryngoscope. 2010;120(5):1011-1021.

9. Stachler RJ, Chandrasekhar SS, Archer SM, Rosenfeld RM, Schwartz SR, Barrs DM, Brown SR, et al. Clinical practice guideline: sudden hearing loss. Otolaryngol Head Neck Surg. 2012;146(3 Suppl):S1-35.

10. Uppal HS, Ayshford CA, Wilson F. Sudden onset bilateral sensorineural hearing loss: a manifestation of occult breast carcinoma. J Laryngol Otol. 2001;115(11):907910.

11. Edwards RL, Rickles FR.Thrombosis and cancer. In Pathophysiology of Haemostasis and Thrombosis. 1984;(7):183-209. 